\title{
Anatomía foliar de Panicum L., Sección Parvifolia (Poaceae, Paniceae) en Venezuela
}

\author{
Lorena I. Guevara $O .^{1}$ \& Mauricio Ramia ${ }^{2}$
}

\begin{abstract}
RESUMEN
(Anatomía foliar de Panicum L., sección Parvifolia (Poaceae, Paniceae) en Venezuela) En el presente trabajo se caracterizó la anatomía foliar de las 10 especies de Panicum sección Parvifolia que presentan mayor dificultad para su delimitación desde el punto de vista morfológico: $P$. arctum, $P$. fonticola, $P$. granuliferum, $P$. micranthum, $P$. pandum, $P$. petrense, $P$. polycomum, $P$. pyrularium, $P$. rivale y $P$. yavitaense, utilizando especimenes provenientes de material de herbario colectados en el sur y centro de Venezuela. Caracteres como: número de células de las extensiones de la vaina vascular, células translúcidas en contacto con la vaina parenquimática y características de los cuerpos de sílice, resultaron ser útiles para la separación de algunas especies. Además se encontraron diferencias significativas en el tamaño de los micropelos bicelulares de la superficie epidérmica en las especies estudiadas.
\end{abstract}

Palabras clave: Poaceae, Panicum, anatomía foliar, Venezuela.

\section{Abstract}

(Leaf anatomy of Panicum L., section Parvifolia (Poaceae, Paniceae) in Venezuela) A characterization of the leaf anatomy using herbarium specimens from the south and center of Venezuela, was carried out on 10 species of Panicum section Parvifolia which are difficult to be distinguished using morphological characters: $P$. arctum, $P$. fonticola, $P$. granuliferum, $P$. micranthum, $P$. pandum, $P$. petrense, $P$. polycomum, $P$. pyrularium, $P$. rivale and $P$. yavitaense. Characters such as the number of cells of the extensions of the vascular sheath, translucent cells in contact with the parenchymatous sheath, and characteristics of silica bodies, proved useful for delimitating some species. Additionally, significant differences in epidermal microhair size were found between studied species.

Key words: Poaceae, Panicum, leaf anatomy, Venezuela.

\section{INTRODUCCIÓN}

El género Panicum L. s. 1., se caracteriza por presentar una panícula abierta o contraída, espiguillas que generalmente desarticulan enteras del pedicelo, con dos flósculos y la primera gluma comúnmente presente. Es uno de los géneros más extensos dentro de la familia Poaceae (Gramineae), con 450 especies de distribución cosmopolita y dentro de este género, la sección Parvifolia se encuentra representada por unas 31 especies (Aliscioni et al. 2003). En Venezuela, la sección Parvifolia está constituida aproximadamente por 19 especies, distribuidas en sabanas húmedas con pocos nutrientes, en morichales y algunas en los tepuyes (M. Ramia, com. pers.).

La sección Parvifolia se considera el grupo más grande y taxonómicamente más dificultoso dentro del género Panicum. Los trabajos realizados sobre este grupo están basados en caracteres cualitativos tales como la duración del ciclo (anual o perenne), forma de la lígula, indumento en las hojas, forma de la espiguilla, presencia y grado de pubescencia, entre otros. Tales caracteres con frecuencia son difíciles de distinguir y parecen no ser consistentes, ya que se observa mucha

\footnotetext{
Artigo recebido em 10/2005. Aceito para publicação em 08/2006.

${ }^{1}$ Universidad Central de Venezuela, Instituto de Botánica Agrícola, Facultad de Agronomía, Apdo. 4573. Vía El Limón, Maracay, Estado Aragua, Venezuela.guevaral@agr.ucv.ve; lorenaguevara@ fastmail.fm

${ }^{2}$ Universidad Central de Venezuela, Fundación Instituto Botánico de Venezuela, Jardín Botánico de Caracas, Apdo. 2156, Caracas, Venezuela.ramiamauricio@hotmail.com

Trabajo financiado por FONACIT y FUNDACITE Aragua, Venezuela.
} 
variabilidad dentro de cada especie (Judziewicz 1990).

Es conocida la importancia que tienen los caracteres anatómicos en la sistemática de las Poaceae siendo, junto a otros rasgos, complemento de los caracteres morfológicos externos. La epidermis de las hojas de las gramíneas exhibe un número de características que son útiles en la identificación o clasificación de los miembros de la familia (Tateoka et al. 1959, Stewart 1965a, Metcalfe \& Clifford 1968). En forma general se ha reportado que la sección Parvifolia incluye especies $\mathrm{C}_{3}$, siendo anatómicamente distinguibles por presentar dos vainas vasculares, la vaina interna del mestoma con células de paredes engrosadas, rodeada por una vaina parenquimática, con células más grandes que las del mesofilo y de paredes delgadas. El número de células del mesofilo entre haces contiguos varía de 5 a 12 y poseen un arreglo irregular (Zuloaga 1987, Zuloaga \& Sendulsky 1988).

Por otra parte, los trabajos sobre anatomía foliar dentro de la sección Parvifolia, son muy escasos, Davidse \& Zuloaga (1991) describieron una especie nueva de la Guayana Venezolana: Panicum tepuianum (Davidse \& Zuloaga), determinando sus afinidades con las secciones Lorea y Parvifolia, usando además de los caracteres morfológicos, algunas características micromorfológicas del flósculo superior, como la presencia y forma de los micropelos bicelulares. Asimismo, determinaron afinidades con $P$. nervosum (Davidse \& Zuloaga) de la sección Parvifolia por medio de características de la anatomía foliar, tales como las extensiones de la vaina vascular parenquimática, la anatomía $\mathrm{C}_{3}$ y la disposición de las células del mesofilo.

Debido a la dificultad que existe para delimitar algunas de las especies de la sección Parvifolia de Venezuela por medio de caracteres morfológicos, en el presente trabajo se realizó la caracterización de la anatomía de la lámina foliar de 10 especies de Panicum L.: $P$. arctum Swallen, $P$. fonticola Swallen, $P$. granuliferum Kunth, $P$. micranthum Kunth, $P$. pandum Swallen, $P$. petrense Swallen, $P$. polycomum Trin., P. pyrularium Hitch. \& Chase, $P$. rivale Swallen y $P$. yavitaense Swallen, con el fin de contribuir a la mejor comprensión de esta sección.

\section{Materiales y Metodos}

El material vegetal estudiado fue extraído de muestras herborizadas de holotipos, isotipos o colecciones identificadas en obras publicadas sobre la sección Parvifolia, procedentes del herbario VEN de la Fundación Instituto Botánico de Venezuela (UCV) y del herbario US del Smithsonian Institution, Washington, USA (Tab. 1).

Se tomó la porción media de la lámina foliar de la hoja que se encuentra debajo de la hoja bandera, siendo esta última la localizada más próxima a la inflorescencia. Estas porciones foliares se rehidrataron parcialmente siguiendo la técnica de preparación de material herborizado para el estudio anatómico mediante el uso de lactofenol de Peña \& Saralegui (1982).

Las porciones foliares parcialmente hidratadas con lactofenol, fueron seccionadas a mano alzada. Para su completa rehidratación, estas secciones se colocaron en una solución acuosa de hipoclorito de sodio al 5\%, durante 20 seg. a 1 min., dependiendo del material. Luego de este tiempo las secciones foliares se enjuagaron exhaustivamente con agua destilada. Para la tinción se utilizó azul de toluidina acuosa $(0.01 \%)$. Se prepararon láminas semipermanentes, montando las secciones foliares en una solución de glicerinaagua $(\mathrm{V}: \mathrm{V})$, sobre láminas portaobjeto.

Para el estudio epidérmico se realizó un aclarado de la lámina foliar colocándose trozos de hoja de aproximadamente $1 \mathrm{~cm}^{2}$ (incluyendo los márgenes foliares), en una solución acuosa de hipoclorito de sodio al $5 \%$, durante 25 minutos a temperatura ambiente. Luego, estos trozos se lavaron con agua destilada, para después teñir con azul de toluidina acuosa al 0,01\% y obtener láminas semipermanentes.

Las láminas fueron observadas y analizadas con un microscopio óptico marca Nikon, utilizando como muestra tres secciones 
Tabla 1 - Material vegetal utilizado en el estudio de especies de Panicum sección Parvifolia

\begin{tabular}{lllll}
\hline Especie & Colector & N $^{\circ}$ Col. & Lugar de colección & Herbario \\
\hline Panicum arctum Swallen & Maguire & 24214 & Surinam & US (Tipo) \\
Panicum fonticola Swallen & Maguire, Cowan \& Wurdak & 29636 & Edo. Amazonas & US (Tipo) \\
Panicum granuliferum Kunth & Curran & 113 & Edo. Amazonas & VEN \\
& Maguire, Wurdak \& Maguire & 41576 & Edo. Amazonas & VEN \\
& Steyermark & 75237 & Edo. Bolívar & VEN \\
Panicum micranthum Kunth & Tamayo & 3995 & Edo. Guárico & VEN \\
& Parra & $\mathrm{s} / \mathrm{n}$ & Edo. Bolívar & VEN \\
& Ortiz \& Ramia & 2617 & Edo. Guárico & VEN \\
Panicum pandum Swallen & Panier \& Schwabe & 19097 & Edo. Bolívar & VEN \\
& Liesner \& Holst & 20508 & Edo. Bolívar & VEN \\
& Liesner \& Holst & 20520 & Edo. Bolívar & VEN \\
Panicum petrense Swallen & Maguire & 29259 & Edo. Amazonas & US (Tipo) \\
Panicum polycomum Trin. & Steyermark & 90472 & Edo. Amazonas & VEN \\
& Steyermark \& Dunsterville & 113131 A & Edo. Bolívar & VEN \\
& Steyermark & 90335 & Edo. Amazonas & VEN \\
Panicum pyrularium Hitch. \& Chase & Liesner \& Delascio & 22209 & Edo. Amazonas & VEN \\
& Steyermark, Holst \& Manara & 131234 & Edo. Bolívar & VEN \\
Panicum rivale Swallen & Guanchez & 371 & Edo. Amazonas & VEN \\
Panicum yavitaense Swallen & Davidse \& Miller & 27235 & Edo. Amazonas & VEN \\
\hline
\end{tabular}

diferentes por espécimen y analizándose en cada una cinco campos visuales. Se realizaron fotografías de las secciones transversales y de las vistas paradérmicas con un equipo microscópico Biomed Leitz con cámara incorporada. La caracterización se realizó siguiendo la terminología sugerida por Ellis (1976, 1979) y Metcalfe (1960). Además, se realizaron ilustraciones de los micropelos bicelulares y cuerpos de sílice para cada uno de los especímenes, utilizando una cámara clara adaptada a un microscopio óptico.

Por otra parte, con el uso de un ocular micrometrado se efectuaron las siguientes mediciones a los micropelos bicelulares, siguiendo a Tateoka et al. 1959: ancho máximo del micropelo (AM), largo de la célula apical (LA), largo de la célula basal (LB), se calculó el índice de longitud de micropelo (LA/LB) tomándose en cuenta 30 repeticiones para cada especie. Los datos obtenidos no cumplieron con el supuesto de normalidad de los errores, sin embargo la población fue lo suficientemente grande para aplicar el Teorema del Límite Central y así aplicar otras pruebas. Con el fin de demostrar si existían diferencias significativas en los valores obtenidos del índice LA/LB y de los valores individuales LA y LB, se aplicó el análisis de varianza de una vía y la prueba de comparaciones múltiples de SNK, utilizándose los siguientes paquetes estadísticos: SAS ver. 8.02 (SAS Institute Inc. 1999-2001) y Statistix ver. 7.0 (Anónimo 2000)

\section{Resultados y Discusión Anatomía foliar}

En la Tabla 2 se resumen las características más resaltantes encontradas en la sección transversal y vista paradérmica de las especies estudiadas.

\section{Secciones Transversales}

En sección transversal, el contorno de la lámina foliar de todas las especies se muestra ligeramente ondulado, generalmente con surcos pequeños en la cara adaxial de la epidermis que se encuentra entre los haces vasculares, encontrándose las células buliformes en esa zona (Figs. 1, 2 y 3 ).

El tejido epidérmico posee una cutícula delgada y presenta muy pocos rasgos diferenciales entre las especies, encontrándose papilas de base ancha, a excepción de $P$. granuliferum, $P$. micranthum y $P$. polycomum en donde están ausentes. Las células buliformes adaxiales están en grupos 
de 3-8 (Figs. 1, 2 y 3). Los macropelos poseen base bulbosa, constricta y las células asociadas a la base tienen forma inflada, sobresaliendo del nivel de la superficie epidérmica (Fig. 3).

El mesofilo presenta arreglo del clorénquima incompletamente radiado, encontrándose de 4 a 12 células clorenquimáticas entre haces vasculares (Fig. 2). Las secciones muestran un solo haz vascular medio (Fig. 1), muy raras veces se observan haces vasculares de 2do orden tal como los describe Ellis (1979). $P$. pandum y $P$. pyrularium generalmente presentan células traslúcidas en contacto y rodeando el haz vascular medio (Fig. 5), las cuales no fueron evidenciadas por Davidse \& Zuloaga (1991) al describir la especie Panicum tepuianum, incluida dentro de la sección Parvifolia. La semilámina consta de 1 a 4 haces vasculares de 1er orden entre los cuales pueden haber de 1 a 3 haces vasculares

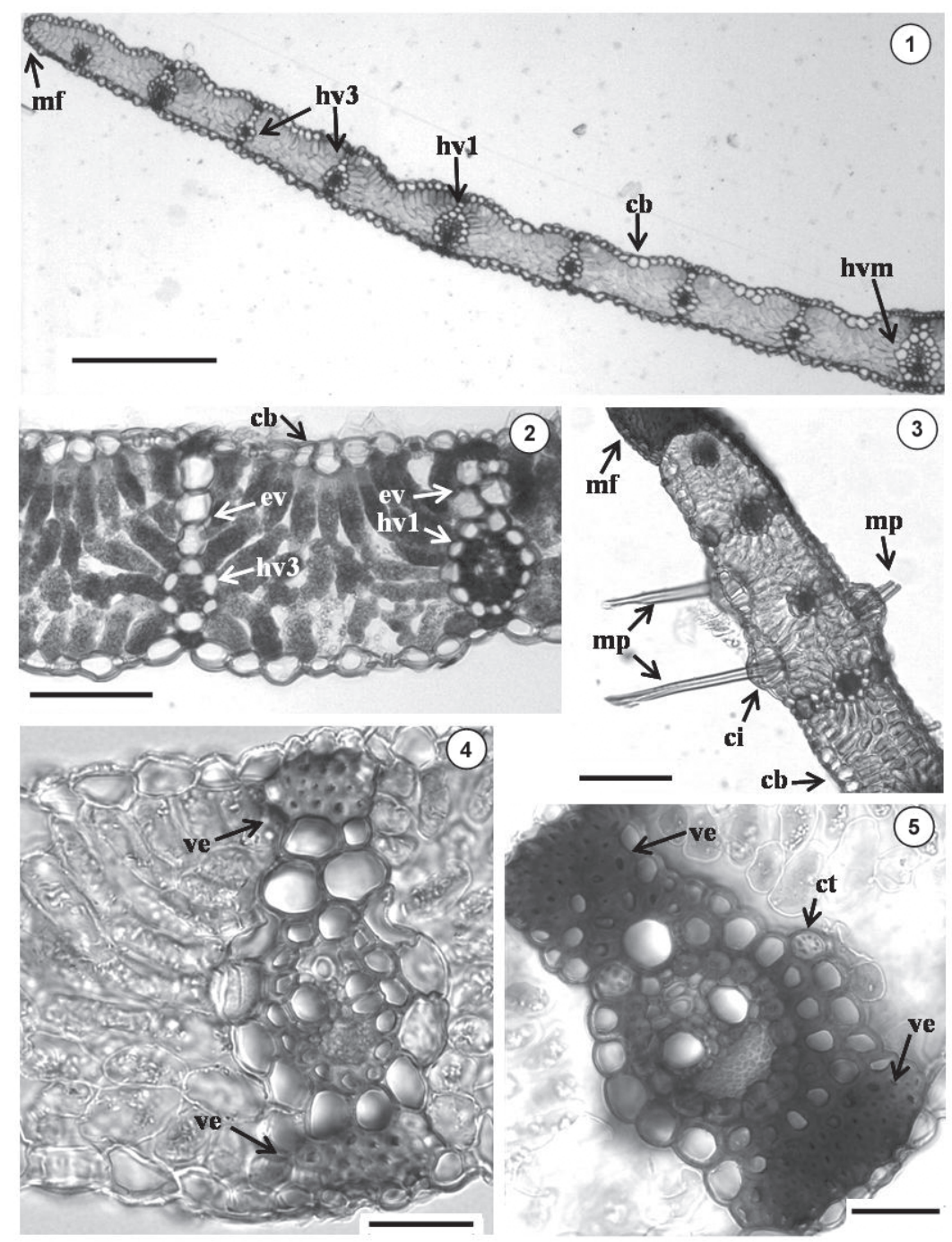

Figura 1.- $P$. fonticola. Vista general de la sección transversal foliar. Figura 2.- P. arctum. Semilámina en sección transversal. Figura 3.- $P$. granuliferum. Semilámina en sección transversal. Figura 4.- $P$. polycomun. Sección tranversal mostrando haz vascular medio. Figura 5.- $P$. pyrularium. Sección transversal mostrando haz vascular medio. Figura 1, barra $=300 \mu \mathrm{m}$; Figuras 2 y 3 , barra $=100 \mu \mathrm{m}$. Figuras 4 y 5 , barra $=30 \mu \mathrm{m}$. (cb) células buliformes; (ci) células infladas asociadas a base de micropelo; (ct) células traslúcidas en contacto con vaina parenquimática; (ev) extensión de la vaina; (hv1) haz vascular de 1er orden; (hv3) haz vascular de 3er orden; (hvm) haz vascular medio; (mf) margen foliar; (mp) macropelo; (ve) viga esclerenquimática. 
de 3er orden, todos ubicados hacia la epidermis abaxial (Fig. 1).

Las vainas vasculares son circulares o elípticas, se encuentran dos en los haces de 1 er orden y una en los de 3er orden. La vaina externa es denominada parenquimática y la interna de mestoma (Brown 1975). La vaina parenquimática de todos los haces vasculares, está compuesta por células parenquimáticas globosas y traslúcidas, con paredes fuerte o ligeramente engrosadas (Figs. 4 y 5), en ocasiones interrumpidas por las prolongaciones de los paquetes de esclerénquima en el haz medio (Fig. 5), siendo estas características coincidentes con lo descrito por Davidse \& Zuloaga (1991) para Panicum tepuianum. Esta vaina presenta extensiones tal como lo señala Renvoize (1987) para las Paniceae, las cuales están conformadas por células de parénquima que se encuentran hacia la cara adaxial de la epidermis (Figs. 1 y 2), únicamente $P$. granuliferum y $P$. micranthum las presentan hacia ambas caras. Casi todas las especies mostraron hasta tres células formando la extensión adaxial de la vaina parenquimática, a excepción de $P$. pyrularium que presentó hasta cuatro.

El tejido esclerénquimático está asociado a los haces vasculares, extendiéndose hacia ambas caras de la epidermis formando vigas esclerenquimáticas (Figs. 4 y 5), también se localiza en los márgenes foliares (Fig. 3). El esclerénquima es abundante en la mayoría de los casos, lo cual se explica como una adaptación al hábitat donde se encuentran estas especies (Lindorf et al. 1991), caracterizado predominantemente por suelos pobres en nutrientes, con saturación de agua o muy secos. El tejido esclerenquimático asociado a los haces vasculares de la semilámina es invariablemente poco desarrollado, a diferencia del esclerénquima del haz vascular medio y el del margen foliar. En la mayoría de los casos estudiados se evidencia poco desarrollo del esclerénquima asociado al margen foliar y mayor desarrollo en el haz vascular medio. Sin embargo, $P$. granuliferum y $P$. micranthum presentaron abundante esclerénquima en ambas regiones. P. arctum mostró poco desarrollo de este tejido en las mismas (Tab. 2).

Las especies estudiadas pertenecen al grupo de las gramíneas $\mathrm{C}_{3}$ o no-Kranz, tal como se ha señalado para el subgénero Phanopyrum (Zuloaga \& Sendulsky 1988, Davidse \& Zuloaga 1991, Aliscioni et al. 2003). Esta condición se evidencia por la presencia de haces vasculares de 1er orden con dos vainas; entre éstas, la vaina de mestoma compuesta por células más engrosadas que las de la vaina parenquimática (Metcalfe 1960, Brown 1975, Renvoize 1987, Zuloaga \& Sendulsky 1988; Lindorf et al. 1991). Asimismo, la presencia de $4-12$ células clorenquimáticas entre haces vasculares adyacentes (Zuloaga 1987), el consecuente amplio espaciado entre estos haces y las células clorenquimáticas incompletamente radiadas, constituyen características que también identifican a las especies como $\mathrm{C}_{3}$ o no-Kranz, como lo indicaron Hattersley \& Watson (1975).

\section{Vistas Paradérmicas}

La cara abaxial de la epidermis presenta constancia en las características y mayor número de caracteres informativos que la cara adaxial, siendo de mayor utilidad la primera para fines descriptivos; esto se corresponde con lo señalado por Stewart (1965a). Sin embargo, la epidermis adaxial fue de particular utilidad para la observación de caracteres como los macropelos y las células buliformes.

Las células largas intercostales poseen paredes anticlinales paralelas, moderadamente engrosadas y con ondulación ligera a moderada en la cara adaxial de la epidermis (Fig. 8). En la cara abaxial pueden ser acortadas (ligeramente más largas que anchas), con las paredes anticlinales paralelas, arqueadas o angulares, moderadamente engrosadas y con ondulación de ligera a profunda (Figs. 7, 9 y 10). P. fonticola y $P$. pandum presentan papilas (pared celular periclinal externa ligeramente convexa) 
constituyendo las células largas costales e intercostales (Fig. 11). Se pueden encontrar papilas hacia los bordes de las zonas intercostales y hacia los estomas (Fig. 11), lo cual se ha reportado como protección al poro estomático (Metcalfe 1960). Presencia de aguijones solo en el margen foliar (Fig. 6), ausentes en $P$. arctum, $P$. fonticola y $P$. rivale. Los macropelos unicelulares son largos, de consistencia dura y de paredes engrosadas generalmente, distribuidos solamente en zonas intercostales, más frecuentes en la epidermis adaxial. En $P$. pandum, los macropelos se distribuyen irregularmente.

El aparato estomático con células subsidiarias predominantemente de tipo domo bajo (Figs. 9, 10 y 11), aunque también se observaron las de tipo triangular en $P$. granuliferum (Fig. 8) y P. polycomum (Fig. 7). La forma de domo bajo y triangular de las células subsidiarias son caracteres que han sido ampliamente reportados tanto para el género
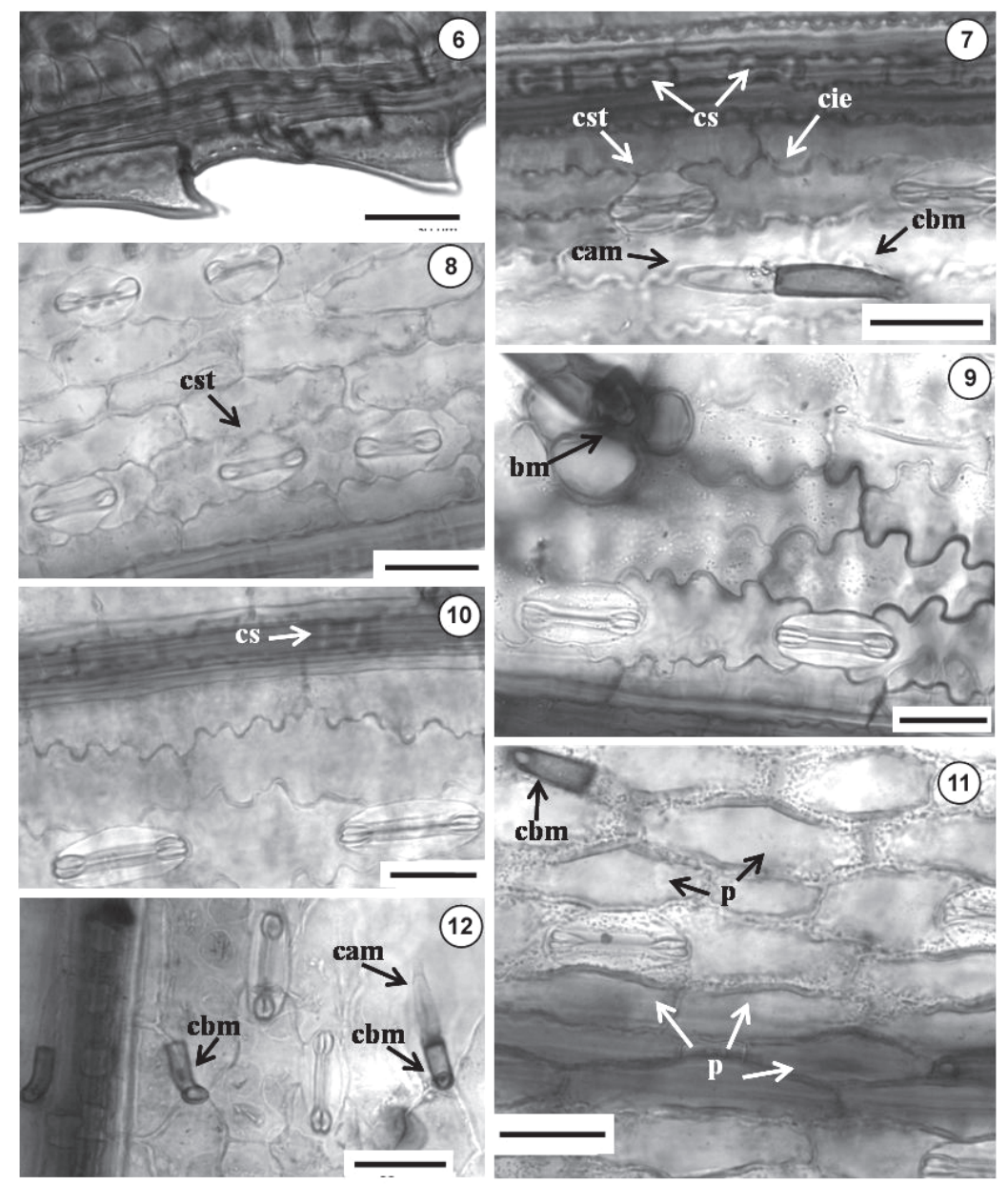

Figura 6 - $P$. pandum. Aguijones en margen foliar. Figura 7 - $P$. polycomum. Vista paradérmica abaxial, zona intercostal (abajo) y costal (arriba). Figura 8 - $P$. granuliferum. Vista pardérmica adaxial, zona costal (abajo) y zona intercostal (arriba). Figura 9 - P. yavitaense. Vista pardérmica abaxial, zona costal. Figura 10 - P. arctum. Vista paradérmica abaxial, zona costal (arriba) y zona intercostal (abajo). Figura 11 - P. fonticola. Vista paradérmica abaxial, zona costal (abajo) y zona intercostal (arriba). Figura 12 - P. micranthum. Vista paradérmica abaxial, zona intercostal con micropelos y estomas. Barras = $30 \mu \mathrm{m}$. (bm) base del macropelo; (cam) célula apical del micropelo; (cbm) célula basal del micropelo; (cie) células interestomáticas; (cs) célula silícica; (cst) célula subsidiaria triangular; (mp) macropelo; (p) papila (pared periclinal externa convexa). 
Panicum como para la subfamilia Panicoideae (Metcalfe 1960, Stewart 1965b, Ellis 1987, Renvoize 1987, Davidse \& Zuloaga 1991). Los estomas se encuentran distribuidos en cada zona intercostal en dos, rara vez tres o cuatro filas longitudinales, ubicados hacia las zonas costales y separadas por más de dos filas de células largas (Fig. 8). Las paredes de las células interestomáticas en contacto con el estoma son cóncavas, en número de 1 , a veces 2 o 3 entre estomas consecutivos. Estas células son cortas en relación al aparato estomático, sólo $P$. polycomum las presenta largas (Fig. 7).

Las células cortas silicificadas se encuentran principalmente en las zonas costales (Figs. 7 y 10), distribuidas en dos a tres filas longitudinales, lo cual está de acuerdo con lo señalado por Metcalfe (1960) como carácter diagnóstico para el género. No obstante, estas células también se encontraron hacia el margen foliar y pocas en las zonas intercostales. Todas las especies poseen células silicificadas con cuerpos de sílice halteriformes, $P$. fonticola, $P$. pandum y $P$. rivale además los presentan nodulares (Figs. 13 b, e e i). Los bordes de los cuerpos de sílice son rectos, indentados o redondeados, siendo esto coincidente con lo señalado por Palmer \& Gerbeth-Jones (1986), para Panicum trichoides $\mathrm{Sw}$. Los cuerpos de sílice son horizontalmente alargados, tal como lo señalado por Ellis (1987) para la subfamilia Panicoideae. Adicionalmente se hallaron cuerpos de sílice equidimensionales en $P$. granuliferum, $P$. micranthum, $P$. petrense y $P$. yavitaense (Figs. $13 \mathrm{c}, \mathrm{d}, \mathrm{f}, \mathrm{j})$. Se observan diferencias en el tamaño y forma de los cuerpos de sílice entre las especies estudiadas. P. pandum, posee cuerpos de sílice conspicuamente más alargados y con forma nodular diferentes a las observadas en el resto de las especies (Fig. 13 e). Igualmente se evidencian similitudes entre algunos de ellos en relación a la forma y el tamaño: $P$. fonticola con $P$. rivale (Figs. 13 b, i); $P$. granuliferum con $P$. petrense (Figs. $13 \mathrm{c}$, f) y $P$. arctum con $P$. yavitaense (Fig. 13 a, j).

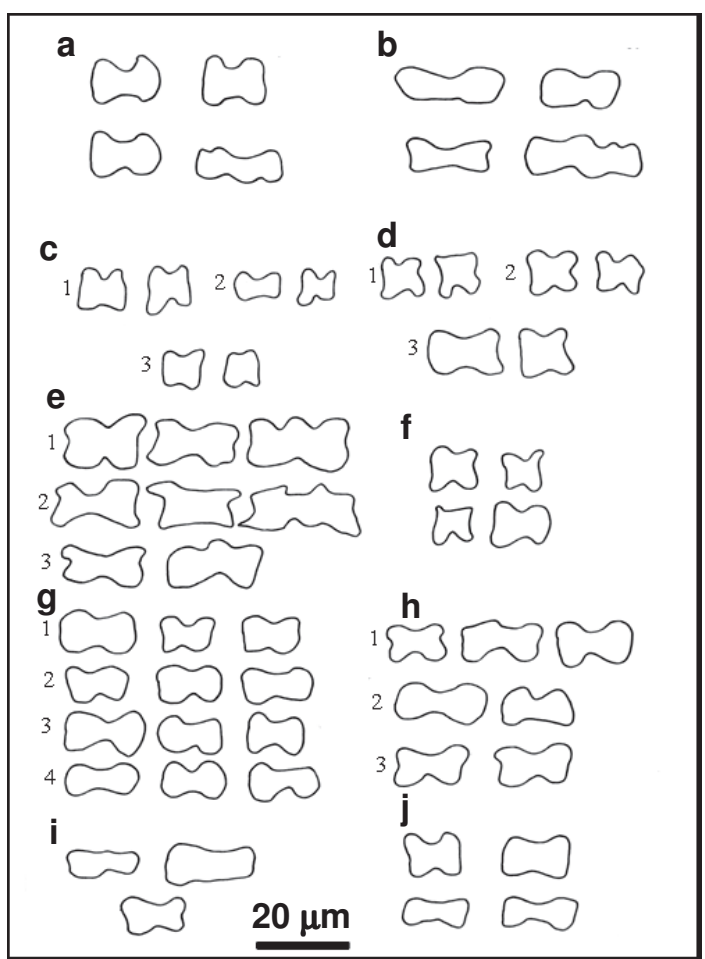

Figura 13 - Forma de los cuerpos de sílice en epidermis foliar de especies de la sección Parvifolia. a. P. arctum Swallen (Maguire 24214). b. P. fonticola Swallen (Maguire, Cowan \& Wurdack 29696). c. P. granuliferum Kunth (1. Steyermark 75237; 2. Maguire, Wurdak \& Maguire 41576; 3. Curran 113). d. P. micranthum Kunth (1. Tamayo 3995; 2. Parra s/n; 3. Ortiz \& Ramia 2617). e. P. pandum Swallen (1. Panier \& Schwabe 19097; 2. Liesner \& Holst 20508; 3. Liesner \& Holst 20520). f. P. petrense Swallen (Maguire 29259). g. P. polycomum Trin. (1. Steyermark 90335; 2. Steyermark 90472; 3. Steyermark \& Dunsterville 113131A; 4. Liesner \& Delascio 22209). h. P. pyrularium Hitch. \& Chase (1. Guanchez 371;2. Steyermark, Holst \& Manara 131234; 3. Davidse \& Miller 27235).i. P. rivale Swallen (Maguire 24732). j. P. yavitaense Swallen (Wurdack \& Adderley 42910).

\section{Micropelos - Análisis Estadístico de las Longitudes}

Están conformados por una célula apical y una basal. La célula apical es caediza, colapsable, de paredes muy delgadas y ápice agudo, mientras que la célula basal tiene paredes engrosadas y base en forma de cúpula (Figs. 7 y 11 y 12). Estos micropelos emergen derechos (rectos); se presentan principalmente en zonas intercostales y hacia el margen foliar, encontrándose pocos en las zonas costales. Las células de inserción de los micropelos son redondeadas o cuadrangulares (Fig. 14). 
Tabla 2 - Resumen de los principales caracteres anatómicos foliares de las especies estudiadas del género Panicum sección Parvifolia.

\begin{tabular}{|c|c|c|c|c|c|c|c|c|c|c|c|}
\hline & Caracteres Anatómicos / Especies & 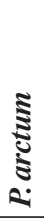 & 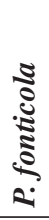 & 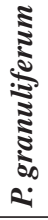 & 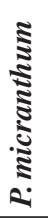 & 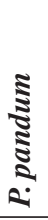 & 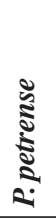 & 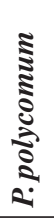 & 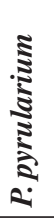 & 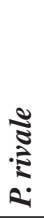 & 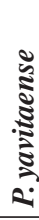 \\
\hline \multirow{17}{*}{ 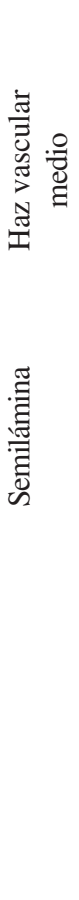 } & Sección transversal & & & & & & & & & & \\
\hline & Mayor desarrollo esclerénquima & & $\mathrm{x}$ & $\mathrm{x}$ & $\mathrm{x}$ & $\mathrm{x}$ & & $\mathrm{x}$ & $\mathrm{x}$ & $\mathrm{x}$ & $\mathrm{x}$ \\
\hline & Interrupción abaxial de la vaina parenquimática & $\mathrm{x}$ & $\mathrm{x}$ & $\mathrm{x}$ & $\mathrm{X}$ & $\mathrm{x}$ & $\mathrm{X}$ & $\mathrm{x}$ & $\mathrm{x}$ & $\mathrm{x}$ & $\mathrm{X}$ \\
\hline & Interrupción adaxial de la vaina parenquimática & & & $\mathrm{x}$ & $\mathrm{x}$ & $\mathrm{X}$ & $\mathrm{X}$ & & & $\mathrm{x}$ & $\mathrm{X}$ \\
\hline & Extensión adaxial de la vaina parenquimática & $\mathrm{x}$ & $\mathrm{x}$ & & & $\mathrm{x}$ & $\mathrm{X}$ & $\mathrm{x}$ & $\mathrm{x}$ & & \\
\hline & Células traslúcidas en contacto con la vaina vascular & & & & & $\mathrm{x}$ & & & $\mathrm{x}$ & & \\
\hline & Vaina vascular en haces de 3er orden con extensión abaxial & & & $\mathrm{x}$ & $\mathrm{x}$ & & & & & & \\
\hline & Células conformando la extensión adaxial en haces de 3er orden de 1-4 & & & & & & & & $\mathrm{x}$ & & \\
\hline & Presencia de macropelos & & $\mathrm{x}$ & $\mathrm{x}$ & $\mathrm{x}$ & $\mathrm{x}$ & & & $\mathrm{x}$ & $\mathrm{x}$ & $\mathrm{x}$ \\
\hline & Vista paradérmica & & & & & & & & & & \\
\hline & Cuerpos de sílice equidimensionales y alargados & & & $\mathrm{x}$ & & & $\mathrm{X}$ & & & & $\mathrm{x}$ \\
\hline & Cuerpos de sílice únicamente equidimensionales & & & & $\mathrm{x}$ & & & & & & \\
\hline & Células interestomáticas relativamente largas & & & & & & & $\mathrm{X}$ & & & \\
\hline & Células subsidiarias tipo triangular & & & $\mathrm{x}$ & & & & $\mathrm{X}$ & & & \\
\hline & Papilas abundantes en la epidermis abaxial & & $\mathrm{x}$ & & & $\mathrm{x}$ & & & & & \\
\hline & Presencia de aguijones en el margen foliar & & & $\mathrm{x}$ & $\mathrm{X}$ & $\mathrm{x}$ & $\mathrm{x}$ & $\mathrm{x}$ & $\mathrm{x}$ & & $\mathrm{x}$ \\
\hline & $\begin{array}{l}\text { Micropelos bicelulares con célula apical de } \\
\text { mayor tamaño que la célula basal }\end{array}$ & & & $\mathrm{X}$ & $\mathrm{X}$ & & $\mathrm{x}$ & & $\mathrm{X}$ & & \\
\hline
\end{tabular}

El largo promedio total de los micropelos fue de $44,82 \mu \mathrm{m}$, lo que coincide con lo indicado por Steward (1965b), para Panicum, siendo además los valores para el largo de la célula apical de los micropelos en este género, similares a los encontrados por Watson \& Dallwitz (1992). El promedio obtenido del ancho máximo de los micropelos (Tab. 6) se acerca al valor de 5,4-6,6 $\mu \mathrm{m}$ indicado por Watson \& Dallwitz (1992). El valor del índice LA/LB promedio de las especies estudiadas fue de 1,22 (Tab. 6), encontrándose dentro del rango de $0,801-1,301 \mu \mathrm{m}$, determinado por Tateoka et al. (1959) para el género Panicum.

Se encontraron diferencias significativas para LA/LB, LA y LB $(a=0,05)$. Al aplicar la prueba al índice de longitud de micropelo (LA/LB) se obtuvo una agrupación diferente a la obtenida cuando se aplicó a las longitudes individuales (Tabs. 3, 4y 5). Además, el coeficiente de variación y la desviación estándar para LA/LB fueron bajos, a diferencia de los encontrados en los valores individuales de LB y LA (Tab. 6), indicando que los valores absolutos de longitudes pueden ser variables y estar posiblemente influenciados por otros factores como los ambientales.

Las especies cuyos micropelos presentaron célula basal más pequeña que la apical fueron: $P$. granuliferum, $P$. micranthum, $P$. petrense y $P$. pyrularium, el resto posee células de longitudes similares. $P$. micranthum es la especie con mayor diferencia en las longitudes de las células de los micropelos y forma un subgrupo aparte. $P$. polycomum fue la especie que presentó menor diferencia en cuanto a las longitudes de las células de los micropelos. El hecho de haberse encontrado diferencias altamente significativas en los valores del índice LA/LB para las 
especies estudiadas, hace inferir que la longitud de las células de los micropelos puede ser útil taxonómicamente a nivel interespecífico, corroborando la pequeña variación intragenérica encontrada en el género Panicum por Tateoka et al. (1959).

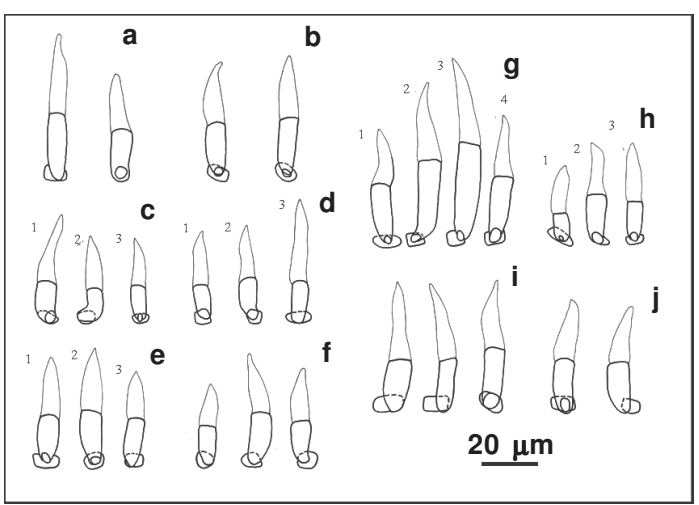

Figura 14 - Micropelos bicelulares en epidermis foliar de especies de la sección Parvifolia. a. P. arctum Swallen (Maguire 24214). b. P. fonticola Swallen (Maguire, Cowan \& Wurdack 29696). c. P. granuliferum Kunth (1. Steyermark 75237; 2. Maguire, Wurdak \& Maguire 41576; 3. Curran 113). d. P. micranthum Kunth (1. Tamayo 3995; 2. Ortiz \& Ramia 2617; 3. Parra s/n). e. P. pandum Swallen (1. Panier \& Schwabe 19097; 2. Liesner \& Holst 20508; 3. Liesner \& Holst 20520).f. P. petrense Swallen (Maguire 29259). g. P. polycomum Trin. (1. Steyermark 90335; 2. Steyermark \& Dunsterville 113131A; 3. Steyermark 90472; 4. Liesner \& Delascio 22209). h. P. pyrularium Hitch. \& Chase (1. Guanchez 371; 2. Steyermark, Holst \& Manara 131234; 3. Davidse \& Miller 27235). i. P. rivale Swallen (Maguire 24732).

j. P. yavitaense Swallen (Wurdack \& Adderley 42910).

Tabla 3 - Prueba de Comparaciones Múltiples de SNK para el largo de la célula basal (LB) de micropelos presentes en la lámina foliar de especies de Panicum sección Parvifolia $(\mathrm{a}=0,05)$

\begin{tabular}{clll}
\hline Agrupamiento & $\mathbf{L B}(\boldsymbol{\mu m})$ & Especie \\
\hline & A & 30,75 & P. polycomum \\
& B & 23,71 & P. arctum \\
& C & 21,66 & P. fonticola \\
& C & 20,71 & P. pandum \\
D & C & 20,34 & P. yavitaense \\
D & C & 20,34 & P. rivale \\
D & E & 18,45 & P. petrense \\
& E & 17,29 & P. pyrularium \\
& E & 16,59 & P. micranthum \\
& F & 14,86 & P. granuliferum \\
\hline
\end{tabular}

Tabla 4 - Prueba de Comparaciones Múltiples de SNK para el largo de la célula apical (LA) de micropelos presentes en la lámina foliar de especies de Panicum sección Parvifolia $(\mathrm{a}=0,05)$.

\begin{tabular}{clll}
\hline Agrupamiento & LA $(\boldsymbol{\mu m})$ & Especie \\
\hline & A & 30,3 & P. polycomum \\
& B & 27,13 & P. arctum \\
& B & 25,73 & P. micranthum \\
C & B & 24,7 & P. rivale \\
C & B & 24,41 & P. pyrularium \\
C & B & 24,29 & P. petrense \\
C & & 22,64 & P. pandum \\
C & & 22,44 & P. fonticola \\
C & & 22,07 & P. yavitaense \\
& D & 19,76 & P. granuliferum \\
\hline
\end{tabular}

Tabla 5 - Prueba de Comparaciones Múltiples de SNK para el índice de longitud de micropelos (LA/LB) presentes en la lámina foliar de especies de Panicum sección Parvifolia $(\mathrm{a}=0,05)$

\begin{tabular}{cccl}
\hline Agrupamiento & LA/LB & Especie \\
\hline & A & 1,57 & P. micranthum \\
& B & 1,44 & P. pyrularium \\
& C & 1,33 & P. petrense \\
& C & 1,33 & P. granuliferum \\
& D & 1,23 & P. rivale \\
E & D & 1,15 & P. arctum \\
E & F & 1,1 & P. pandum \\
E & F & 1,09 & P. yavitaense \\
G & F & 1,03 & P. fonticola \\
G & & 0,98 & P. polycomum \\
\hline
\end{tabular}

Tabla 6 - Valores de medias, coeficiente de variación y desviación estándar de las longitudes de las células de los micropelos bicelulares presentes en la lámina foliar de especies de Panicum sección Parvifolia. AM: ancho máximo; LB: longitud célula basal; LA: longitud célula apical.

\begin{tabular}{lllll}
\hline & \multicolumn{4}{c}{ Variable } \\
\hline Estadístico & AM & LB & LA & LA/LB \\
Media & $7,89 \mu \mathrm{m}$ & $20,47 \mu \mathrm{m}$ & $24,35 \mu \mathrm{m}$ & 1,22 \\
Coef. variación & 10,73 & 15,32 & 16,85 & 13,47 \\
Desv. estándar & 0,93 & 5,23 & 4,91 & 0,24 \\
\hline
\end{tabular}




\section{Conclusiones}

La anatomía foliar de las especies estudiadas del género Panicum sección Parvifolia es homogénea, sin embargo se encontraron algunos caracteres que resultaron ser útiles con fines taxonómicos para algunas de las especies.

La presencia de células traslúcidas redeando la vaina parenquimática del haz medio junto a los cuerpos de sílice conspicuamente más alargados en relación a los de las otras especies y de forma nodular, diferencian a $P$. pandum. Las células interestomáticas más largas que el aparato estomático, la presencia de células subsidiarias tipo triangular, micropelos bicelulares con células apical y basal de mayor tamaño que el resto de las especies y de igual tamaño entre sí, son características que separan anatómicamente a $P$. polycomum. Las células traslúcidas rodeando la vaina parenquimática en el haz medio, junto a la presencia de hasta cuatro células en la extensión de la vaina parenquimática distinguen a $P$. pyrularium. Por otra parte, se evidencia que $P$. granuliferum, $P$. micranthum y $P$. petrense constituyen un grupo muy afín, ya que presentan caracteres en común, como un valor del índice LA/LB similar, cuerpos de sílice equidimensionales de forma y tamaño muy similar en las tres especies y poco desarrollo de tejido esclerenquimático en el margen foliar. El resto de las especies: $P$. arctum, $P$. fonticola, $P$. rivale y $P$. yavitaense demostraron ser anatómicamente homogéneas, por lo que desde este punto de vista no es posible separarlas.

Anatómicamente, todas las especies estudiadas pertenecen al grupo de las gramíneas $\mathrm{C}_{3}$ o no-Kranz, ya que poseen dos vainas vasculares, el número de células clorenquimáticas entre haces vasculares contiguos es de 4 hasta 12 y su arreglo es incompletamente radiado.
Las diferencias significativas encontradas en los valores obtenidos del índice de longitud de micropelos (LA/LB) para las especies estudiadas, indica que la longitud de las células de los micropelos puede ser útil taxonómicamente a nivel interespecífico.

\section{Agradecimientos}

A la profesora Emira Fanny de Torres, por su guía y gran apoyo. Al Instituto de Botánica Agrícola y al Postgrado en Botánica Agrícola de la Facultad de Agronomía, Universidad Central de Venezuela, por todo el apoyo logístico ofrecido para la realización de este trabajo, en especial a las profesoras Damelis Jáuregui y Thirza Ruiz. También a FONACIT y a FUNDACITE Aragua, por el apoyo económico.

\section{REFERENCIAS BIBLIOGRÁFICAS}

Aliscioni, S.; Giussani, L.; Zuloaga, F. \& Kellogg, E. 2003. A molecular phylogeny of Panicum (Poaceae: Paniceae): tests of monophyly and phylogenetic placement within the Panicoideae. American Journal Botany 90(5): 796-821.

Anónimo. 2000. Analytical Software. STATISTIX 7.0. Florida.

Brown, W. V. 1975. Variations in anatomy, associations and origins of Kranz tissue. American Journal of Botany 62(4): 395-402.

Davidse, G. \& Zuloaga, F. 1991. Panicum tepuianum (Poaceae: Paniceae), a new species from Cerro Aracamuni in the Venezuelan Guayana. Novon 1: 191-195.

Ellis, R. P. 1976. A procedure for standardizing comparative leaf anatomy in the Poaceae I. The leaf-blade as viewed in transverse section. Bothalia 12(1): 65-109.

1979. A procedure for standardizing comparative leaf anatomy in the Poaceae II. The epidermis as seen in surface view. Bothalia 12(1): 641-671.

1987. A review of comparative leaf blade anatomy in systematics of the 
Poaceae: the past twenty-five years. In: Soderstrom, T.; Hilu, K.; Campbell, C. \& Barkwoth, M. (eds.). Grass systematics and evolution. Smithsonian Institution Press, Washington. Pp. 3-10.

Hattersley, P. W. \& Watson, L. 1975. Anatomical parameters for predicting photosynthetic pathways of grasses leaves: the 'maximun lateral cell count' and the 'maximum cells distant count'. Phytomorphology 25(3): 325-333.

Judziewicz, E. 1990. Poaceae. In: Gürts-Van Rijn, A. R. A. (ed.). The Flora of the Guianas. Koeltz, Koenigstein, 727p.

Lindorf, H.; Parisca, L. \& Rodríguez, P. 1991. Botánica. Clasificación, estructura, reproducción. Ediciones de la Biblioteca, U.C.V., Caracas, 584p.

Metcalfe, C. R. 1960. Anatomy of the Monocotyledons I: Gramineae. Clarendon Press, Oxford, 731p.

\& Clifford, H. 1968. Microhair on grasses. Kew Bulletin. 21(3): 490.

Palmer, P. \& Gerbeth-Jones, S. 1986. A scaning electron microscope survey of the epidermis of east African grasses, IV. Smithsonian Contributions to Botany 62 .

Peña, E. \& Saralegui, H. 1982. Técnicas de anatomía vegetal. Universidad de La Habana, La Habana, 100p.

Renvoize, S. A. 1987. A survey of leaf-blade anatomy in grasses XI: Paniceae. Kew Bulletin 42(3): 739-768.
SAS Institute Inc. 1999-2001. SAS release 8.02. Cary.

Stewart, D. R. 1965a. The epidermal characters of grasses with special reference to east African plains species. $1^{\text {st }}$ Part. Botanische Jahrbücher 84(1): 63-116.

1965b. The epidermal characters of grasses with special reference to east African plains species. $2^{\text {nd }}$ Part. Botanische Jahrbücher 84(2): 117-174.

Tateoka, T.; Sukemitsu, I. \& Kawano, S. 1959. Notes on grasses. IX. Systematic significance of bicellular microhairs of leaf epidermis. Botanical Gazette 121(2): 80-91.

Watson, L. \& Dallwitz, M. J. 1992. Grass genera of the world, [en línea]. Version: 10th August 2003. Dirección URL: $<$ http://delta-intkey.com>. [Consulta: sep.-oct 2003].

Zuloaga, F. O. 1987. Systematics of new world species of Panicum (Poaceae: Paniceae). In: Soderstrom, T.; Hilu, K.; Campbell, C. \& Barkwoth, M. (eds.). Grass systematics and evolution. Smithsonian Institution Press, Washington. Pp: 287-306.

$\&$ Sendulsky, T. 1988. A revision of Panicum subgenus Phanopyrum section Stolonifera (Poaceae: Paniceae). Annals of the Missouri Botanical Garden 75: 420-455. 